\title{
Factors influencing the time to surgery after neoadjuvant chemotherapy in breast cancer patients
}

\author{
Carolin Müller ${ }^{1} \mathbb{C}$ - Ingolf Juhasz-Böss ${ }^{1}$. Gilda Schmidt ${ }^{1}$. Peter Jungmann ${ }^{1}$ - Erich-Franz Solomayer ${ }^{1}$. \\ Georg-Peter Breitbach ${ }^{1}$. Stephanie Juhasz-Böss ${ }^{1}$
}

Received: 20 August 2019 / Accepted: 5 March 2020 / Published online: 14 March 2020

(c) The Author(s) 2020

\begin{abstract}
Purpose It is suspected that delayed surgery after neoadjuvant chemotherapy (NACT) leads to a worse outcome in breast cancer patients. We therefore evaluated possible influencing factors of the time interval between the end of NACT and surgery. Methods All patients receiving NACT due to newly diagnosed breast cancer from 2015 to 2017 at the Department of Gynecology, Saarland University Medical Center, were included. The time interval between end of NACT and surgery was defined as primary endpoint. Possible delaying factors were investigated: age, study participation, outpatient and inpatient presentations, implants/expander, MRI preoperatively, discontinuation of chemotherapy, and genetic mutations.

Results Data of 139 patients was analyzed. Median age was 53 years (22-78). The time interval between end of NACT and surgery was 28 days (9-57). Additional clinical presentations on outpatient basis added 2 days $(p=0.002)$ and on inpatient basis added 7 days to time to surgery $(p<0.001)$. Discontinuation of NACT due to chemotherapy side effects prolonged time to surgery by 8 days $(p<0.001)$, whereas discontinuation due to disease progress did not delay surgery $(p=0.6)$. In contrast, a proven genetic mutation shortened time to surgery by 7 days $(p<0.001)$. Patient's age, participation in clinical studies, oncoplastic surgery, and preoperative MRI scans did not delay surgery.

Conclusion Breast care centers should emphasize a reduction of clinical presentations and a good control of chemotherapy side effects for breast cancer patients to avoid delays of surgery after NACT.
\end{abstract}

Keywords Breast cancer $\cdot$ Neoadjuvant therapy $\cdot$ Time to surgery $\cdot$ Delaying factors

\section{Introduction}

Breast cancer is the most frequent malign disease in women. Lifetime risk adds up to 1:8 [1] and the incidence of breast cancer has even increased over the last years so that in 2020 about 77,600 new diseases may be expected in Germany. Breast cancer mortality has dropped since 1990 thanks to many new successful therapy strategies [1]. One of the therapy strategies is the neoadjuvant chemotherapy (NACT) which allows disease downstaging and higher rates of breastconserving therapy [2]. According to German guidelines, the operation following NACT should be performed $2-4$ weeks after the end of NACT to bypass the leukocyte nadir [3].

Carolin Müller

carolin.mueller@uks.eu

1 Department of Gynecology, Obstetrics and Reproductive Medicine, Saarland University Medical Center, Kirrbergerstraße 100, 66424 Homburg/Saar, Germany
There is compelling evidence that a delayed therapy onset of adjuvant chemotherapy worsen outcome $[4,5]$, which was also shown for neoadjuvant settings [6]. Sanford et al. reported worse outcome when time to surgery exceeds 8 weeks after NACT [6]. Several factors might influence this time interval, and especially prolonging factors are of considerable interest to improve clinical pathways.

We therefore determined the time interval between the end of NACT and surgery, and investigated possible influencing factors: age, study participation, outpatient and inpatient presentations, implants/expander, MRI preoperatively, discontinuation of chemotherapy, and genetic mutations. We previously reported influences on the onset of NACT based on the same register [7]. Onset of NACT was delayed by additional clinical presentations, making it particularly interesting, whether this also applies to the time between NACT and surgery. 


\section{Patients and methods}

\section{Data collection}

Data were collected of all patients receiving a neoadjuvant chemotherapy (NACT) at the Department of Gynecology, Saarland University Medical School from 2015 to 2017. Therapy schedule was based on diagnosis and determination of prognostic factors. The primary endpoint was the time interval between the end of NACT (date of last infusion) and date of surgery.

Patient's age, stage, and tumor biology were recorded. The type of operation was documented (breast-preserving therapy versus mastectomy and reconstruction with breast implants or expander). Chemotherapy plans were based on clinical studies or standard therapy was applied. From 2015 to 2017, the following neoadjuvant study designs were addressed: GAIN II, GeparOcto, GeparX, Nadens, and Adapt studies. The number of presentations in the hospital was recorded, either on outpatient or inpatient basis. It was noted whether there was a genetic mutation or whether additional imaging (for example MRI) had to be performed before surgery. Furthermore, discontinuation of chemotherapy and reasons for discontinuation (therapy complications/disease progress) were recorded.

\section{Data management and statistics}

Patient's data were reviewed in the hospital's digital documentation system (SAP, Walldorf, Germany). Data was collected using Microsoft Excel $2010^{\circledR}$ (Microsoft, Redmond, USA). Further statistics were performed with SPSS 24.0 (IBM, Armonk, USA). Quantitative parameters like time intervals, patient's age, and number of clinical presentations are presented as median and range. Qualitative parameters like tumor biology, type of operation (breastconserving therapy, mastectomy or reconstruction with breast implants or expander), and other influencing factors are given as frequencies. Univariate regression analysis was performed, with bootstrapping of 100,000 samples, to determine the influence of the following factors on the time interval between the end of NACT and surgery: age, study participation, clinical presentations, reconstructive surgery (implants/expander), preoperative MRI, discontinuation of chemotherapy (therapy complications/disease progress), and genetic mutation. All procedures performed in the study involving human participants were in accordance with the ethical standards of the institutional and/or national research committee and with the 1964 Helsinki declaration and its later amendments or comparable ethical standards. Informed consent was obtained from every individual participant included in the study.

\section{Results}

A total of 152 patients were scheduled for NACT between 2015 and 2017. After exclusion of 13 patients with insufficient records, 139 patients proved to be evaluable. Overall median time between the end of NACT and surgery was 28 days (9-57). The patient's median age was 54 years (22-78).

Table 1 illustrates tumor and therapy characteristics. About half of the patients had a complete remission (ypT0) after NACT. None of the patients had primary metastasis. The most common surgical procedure was breast-conserving therapy (65\%). The luminal A subtype was rare $(4 \%)$, whereas luminal B, Her2-positive and triple-negative subtypes were equally distributed $(32,34,30 \%)$. Genetic mutation was rare (4\%). 90 patients $(65 \%)$ were treated with standard chemotherapies according to guidelines and $49(35 \%)$ received study therapies. The major part $(47 \%)$ was treated according to the GAIN II protocol (23 patients) [8] followed by GeparOcto (18 patients) [9]. A total of 27 patients $(19 \%)$ discontinued chemotherapy. Most of the patients discontinued chemotherapy due to side effects (20 patients) and some due to disease progress (4 patients). Therapy complications were polyneuropathy in nine patients, changes of laboratory values (for example increased liver value) in four patients, skin lesions (for example erythema) in three patients, port infection in one patient, and discontinuation of chemotherapy due to general deterioration in three patients.

Between the end of chemotherapy and operation, the patients had a median of 1 (0-7) outpatient presentations and a median of $0(0-2)$ inpatient presentations. The major reasons for clinical presentations were planning of surgery (98\%) followed by side effects of chemotherapy (17\%), which is consistent with main reasons for repetitive appointments due to planning of surgery (29\%) and due to side effects of chemotherapy $(11 \%)$. In rare cases, a preoperative MRI (14\%) or echocardiography (9\%) was performed before operation (Table 2).

Clinical presentations on outpatient and inpatient basis considerably prolonged time to surgery. Every outpatient presentation added 2 days and every inpatient presentation added 7 days to the time to surgery. Discontinuation of NACT due to side effects also prolonged time to surgery by an average of 8 days, whereas discontinuation due to disease progress had no significant influence. A proven 
Table 1 Tumor and therapy characteristics

$\begin{array}{ll}\text { Absolute } & \text { Cumulative } \\ \text { frequencies } & \text { frequencies } \\ (n) & (\%)\end{array}$

\section{Staging}

TNM-stage

ypT

0

1

2

3

4

$\mathrm{X}$

$\mathrm{ypN}$

0

1

2

3

$\mathrm{X}$

$\mathrm{pN}$ (sentinel node)

$$
0
$$

$$
1
$$

$$
\mathrm{X}
$$$$
\text { M }
$$$$
0
$$

\begin{tabular}{|c|c|c|}
\hline Breast-conserving therapy & 90 & 64.7 \\
\hline Subcutaneous mastectomy & 14 & 10.1 \\
\hline Simple mastectomy & 35 & 25.2 \\
\hline Implants/expander & 19 & 13.6 \\
\hline \multicolumn{3}{|l|}{ Histology } \\
\hline \multicolumn{3}{|l|}{ Histological subtype } \\
\hline \multicolumn{3}{|l|}{$\begin{array}{l}\mathrm{ER} \pm, \mathrm{PR} \pm, \text { Her2neu neg, } \\
\mathrm{Ki} 67 \leq 15 \%\end{array}$} \\
\hline \multicolumn{3}{|l|}{$\begin{array}{l}\mathrm{ER} \pm, \mathrm{PR} \pm, \text { Her2neu neg, } \\
\mathrm{Ki} 67>15 \%\end{array}$} \\
\hline $\begin{array}{l}\text { Her2 like } \\
\text { ER } \pm, P R \pm, \text { Her2neu }+\end{array}$ & 47 & 33.8 \\
\hline Triple negative & 41 & 29.5 \\
\hline \multicolumn{3}{|l|}{ Discontinuation of chemotherapy } \\
\hline \multicolumn{3}{|l|}{ Reasons } \\
\hline Side effects of chemotherapy & 20 & 14.3 \\
\hline Disease progress & 4 & 2.9 \\
\hline
\end{tabular}

Surgery

Type of surgery

genetic mutation shortened the time to surgery by an average of 7 days. Age, study participation, oncoplastic surgery (implants / expander), and preoperative MRI did not delay surgery (Table 3).
Table 2 Reasons for clinical presentations between neoadjuvant chemotherapy and surgery

\begin{tabular}{lll}
\hline & $\begin{array}{l}\text { Absolute } \\
\text { frequencies } \\
(n)\end{array}$ & $\begin{array}{l}\text { Cumulative } \\
\text { frequencies } \\
(\%)\end{array}$ \\
\hline
\end{tabular}

Planning of surgery

Number of presentations

$\begin{array}{lrl}1 & 114 & 81.4 \\ 2 & 20 & 14.3 \\ 3 & 1 & 0.7 \\ 4 & 1 & 0.7 \\ 5 & 1 & 0.7\end{array}$

Side effects of chemotherapy

Number of presentations

\begin{tabular}{lrl}
1 & 15 & 10.7 \\
2 & 6 & 4.3 \\
3 & 1 & 0.7 \\
5 & 1 & 0.7 \\
7 & 1 & 0.7 \\
All & 24 & 17.1 \\
Further diagnostics & & \\
Echocardiography & 12 & 8.6 \\
Imaging (MRI, CT) & 19 & 13.6 \\
Main reason for repetitive appointments & & \\
All & 56 & 40 \\
Planning of surgery & 40 & 28.6 \\
Side effects of chemotherapy & 16 & 11.4 \\
\hline
\end{tabular}

Table 3 Influences on time to surgery assessed by univariate regression analysis

\begin{tabular}{lllr}
\hline Parameter & $\begin{array}{l}\text { Regression } \\
\text { coefficient }\end{array}$ & $\begin{array}{l}\text { 95\% confidence } \\
\text { interval }\end{array}$ & $p$ \\
\hline Age & 0.007 & -0.1 to 0.1 & 0.054 \\
$\begin{array}{l}\text { Study participation } \\
\text { Outpatient presenta- }\end{array}$ & 0.5 & -2.4 to 3.5 & 0.72 \\
$\quad$ tions & 2.2 & 0.8 to 3.6 & 0.002 \\
$\begin{array}{l}\text { Inpatient presenta- } \\
\text { tions }\end{array}$ & 6.9 & 3.9 to 10.3 & $<0.001$ \\
$\begin{array}{l}\text { Implants/expander } \\
\text { MRI (preoperative) }\end{array}$ & 3 & -1.4 to 7.6 & 0.189 \\
$\begin{array}{l}\text { Discontinuation of } \\
\text { chemotherapy (side }\end{array}$ & 8.2 & -0.8 to 8.4 & 0.105 \\
$\quad$ effects) & & 3.7 to 12.6 & $<0.001$ \\
$\begin{array}{l}\text { Discontinuation of } \\
\text { chemotherapy (dis- }\end{array}$ & -1 & -5.7 to 2.7 & 0.622 \\
$\quad$ ease progress) & & & $<0.001$ \\
\begin{tabular}{l} 
Genetic mutation \\
\hline
\end{tabular} & -6.6 & -8.8 to -4.5 &
\end{tabular}




\section{Discussion}

Median time between NACT and surgery was 28 days in accordance with current German guidelines which recommend surgery 2-4 weeks after NACT [3]. Similar results were reported in a retrospective analyses of 1101 patients with 33 days between NACT and surgery [6]. Additionally, outcome seems to worsen when surgery is performed more than 8 weeks after NACT [6].

Worse outcomes by a delayed therapy onset was also shown for adjuvant settings. Especially patients with stage III of breast cancer, trastuzumab-treated Her2-positive tumors and triple negative carcinomas had inferior outcomes when time to chemotherapy was longer than 60 days compared to a therapy onset below 30 days after surgery [4]. Consistently, recurrence-free survival and overall survival decreases in patients with triple-negative breast cancer when chemotherapy started more than 30 days after surgery [5]. However, these studies investigated adjuvant chemotherapy settings, where chemotherapy is performed after surgery. There is still missing data for limits of the time between NACT and surgery. However, therapy centers should stick to the recommended range of 2-4 weeks [3], as worse outcome can be expected if surgery is delayed [6].

To provide possible approaches to improve patient care, we analyzed possible influences on time to surgery after NACT. Additional clinical presentations prolonged time to surgery-outpatient presentations by 2 days and inpatient presentations by 7 days. The most common reason for a clinical presentation was planning of surgery. Most of the patients were seen after completion of chemotherapy to plan surgery, but some (2\%) were seen earlier when regular presentations for sonographic controls were already scheduled close to the end of chemotherapy-this is not standard of care but saves time and resources. This might be a considerable approach to minimize clinical presentations. Especially patients with complicated cases could already be advised during clinical follow-ups of chemotherapy. The second most common reason for a clinical presentation were side effects of chemotherapy. A good control of chemotherapy side effect is thus recommendable. For example, by routine antiemetic prophylaxis or GCSF (granulocyte colony stimulating factor) preparations. Taken together, breast care centers should optimize clinical pathways and the prevention of chemotherapy side effects to minimize clinical presentations and accelerate time to surgery.

Patient's age had no influence on time to therapy onset, nor did study participation (Table 3). A possible reason might be that patients participating in clinical studies had the most part of administrative and structural restraints (like checking of inclusion/exclusion criteria, control of morphologic and biologic assessment, etc.) already done before NACT. One might expect that this delays the onset of NACT, but our previous analysis based on the same register showed no delay of NACT by study participation [7].

Oncoplastic surgery did not prolong time to surgery. Oncoplastic surgery offers a further approach for extending breast conserving surgery possibilities, with wide excisions and aesthetic results [10]. Although oncoplastic surgery is performed more frequently in patients with larger or multifocal tumors, there is no increased rate of local recurrences or re-operations [11]. Taken together, there is no reason to forgo oncoplastic surgery in terms of outcome and time to surgery.

Preoperative imaging (MRI scan) seemed to delay time to surgery on average by 4 days without reaching statistical significance (Table 3). This might be due to the limited number of patients. Previous studies reported delays of 11 [12] or even 22 days [13]. However, both studies were done in adjuvant therapy settings. The need for a preoperative MRI might be already clear during NACT, presumably allowing timely arrangements. Nevertheless, available data suggests that structural and organizational conditions should be optimized to minimize a possible delay of surgery by preoperative MRI.

The discontinuation of chemotherapy due to chemotherapy side effects prolonged time to surgery by 8 days. The majority of the patients stopped chemotherapy because of intolerable side effects, whereas a minority had disease progress. Patients suffering from side effects may have had to recover prior to surgery, presumably delaying time to surgery. In contrast, discontinuation of chemotherapy due to disease progress did not delay time to surgery. This seems to be preferable, as patients with disease progress need timely surgical treatment.

Time to surgery was shortened by 7 days, if genetic mutation was confirmed. Since patients with proven BRCA mutation are more likely to develop breast cancer, primary mastectomy is usually advised [14]. Neoadjuvant settings give enough time for genetic counseling and testing, so that the result will already be available before completion of chemotherapy. Thus, the recommended surgical procedure is already clear before NACT is completed, making it apparent that time to surgery is shortened.

Our study has some limitations. This study was performed in a single certified tertiary breast care center. Clinical pathways might differ in other centers. Sentinel-node-biopsy before NACT was standard of care in Germany during the study period. 72 patients (52\%) in this study received SNB prior to NACT. With positive pretherapeutic lymph nodes (sonographic or according to SNB), an axillary lymphadenectomy was added to the surgical treatment after completion of chemotherapy. Nowadays, sentinel-node-biopsy 
is performed after NACT, which may additionally prolong time to surgery. Furthermore, sample size is small, and thus regression analysis had to be performed with bootstrapping. At least, not all possible delaying factors were addressed in this study. For example, some patients might ask for a second opinion before surgery.

\section{Conclusion}

Median time between NACT and surgery is 28 days. To avoid delays of surgery, breast care centers should emphasize a reduction of appointments for clinical presentations of patients with breast cancer after NACT. A good control of chemotherapy side effects is recommendable, as this is the major reason for chemotherapy discontinuation which delays surgery. Earlier clarity about the surgical procedure seems to shorten the time to surgery for patients with proven genetic mutation, thus early planning of surgery should be emphasized. At least, patient's age, participation in clinical studies, oncoplastic surgery, discontinuation of chemotherapy due to disease progress, and preoperative MRI scans seems not to delay surgery.

Acknowledgement Open Access funding provided by Projekt DEAL.

Author contribution All authors contributed to the study conception and design. Material preparation, data collection and analysis were performed by Carolin Müller. The first draft of the manuscript was written by Carolin Müller and all authors commented on previous versions of the manuscript. All authors read and approved the final manuscript.

\section{Compliance with ethical standards}

Conflict of interest All authors declare no conflict of interest.

Ethical approval This study is in accordance with the ethical standards of the institutional and/or national research committee and with the 1964 Helsinki declaration and its later amendments or comparable ethical standards. Trial registration number: 207/10 (Ethics committee of the Saarland Physician's chamber). This article does not contain any studies with animals performed by any of the authors.

Informed consent Informed consent was obtained from every individual participant included in the study.

Open Access This article is licensed under a Creative Commons Attribution 4.0 International License, which permits use, sharing, adaptation, distribution and reproduction in any medium or format, as long as you give appropriate credit to the original author(s) and the source, provide a link to the Creative Commons licence, and indicate if changes were made. The images or other third party material in this article are included in the article's Creative Commons licence, unless indicated otherwise in a credit line to the material. If material is not included in the article's Creative Commons licence and your intended use is not permitted by statutory regulation or exceeds the permitted use, you will need to obtain permission directly from the copyright holder. To view a copy of this licence, visit http://creativecommons.org/licenses/by/4.0/.

\section{References}

1. Koch-Institut R (2016) Bericht zum Krebsgeschehen in Deutschland. Bericht zum Krebsgeschehen Deutschl 2016:16-77

2. Bartsch R, Bergen E, Galid A (2018) Current concepts and future directions in neoadjuvant chemotherapy of breast cancer. MemoMag Eur Med Oncol 11:199-203

3. AGO Breast Committee. (2019) Diagnosis and treatment of patients with primary and metastatic breast cancer. Recommendations. https://www.ago-online.de Accessed 26 Jan 2020

4. De Melo GD, Gonzalez-Angulo AM, Lei X, Theriault RL, Giordano SH, Valero V et al (2014) Clinical impact of delaying initiation of adjuvant chemotherapy in patients with breast cancer. J Clin Oncol 32:735-744

5. Morante Z, Ruiz R, De la Cruz-Ku G, Namuche F, Mantilla $\mathrm{R}$, Lujan $\mathrm{M}$, et al.: Impact of the delayed initiation of adjuvant chemotherapy in the outcomes of triple negative breast cancer. Available from: https://www.ascopost.com/News/59545

6. Sanford RA, Lei X, Barcenas CH, Mittendorf EA, Caudle AS, Valero V et al (2016) Impact of time from completion of neoadjuvant chemotherapy to surgery on survival outcomes in breast cancer patients. Ann Surg Oncol 23:1515-1521

7. Müller C, Juhasz-Böss S, Schmidt G, Solomayer EF, Juhasz-Böss I, Breitbach GP (2019) Factors influencing the onset of neoadjuvant therapy in breast cancer patients. Breast Care. https://doi. org/10.1159/000502223

8. GAIN II, https://clinicaltrials.gov/ct2/show/NCT01690702. Accessed 24 July 2019

9. GeparOcto, https://clinicaltrials.gov/ct2/show/NCT02125344. Accessed 24 July 2019

10. Berry MG, Fitoussi AD, Curnier A, Couturaud B, Salmon RJ (2010) Oncoplastic breast surgery: a review and systematic approach. J Plast Reconstr Aesthet Surg 63:1233-1243

11. Niinikoski L, Leidenius MHK, Vaara P, Voynov A, Heikkilä $P$, Mattson J et al (2019) Resection margins and local recurrences in breast cancer: comparison between conventional and oncoplastic breast conserving surgery. Eur J Surg Oncol 45:976-982

12. Zhang M, Sun S, Mesurolle B (2017) The impact of pre-operative breast MRI on surgical waiting time. PLoS ONE 12:1-10

13. Bleicher RJ, Ciocca RM, Egleston BL, Sesa L, Evers K, Sigurdson ER et al (2009) Association of routine pretreatment MRI with time to surgery, mastectomy rate, and margin status. J Am Coll Surg 209:1-13

14. Song CV, Teo SH, Taib NA, Yip CH (2018) Surgery for BRCA, TP53 and PALB2: a literature review. Ecancermedicalscience 12:1-10

Publisher's Note Springer Nature remains neutral with regard to jurisdictional claims in published maps and institutional affiliations. 